\title{
Symmetry control of radiative decay in linear polyenes: Low barriers for isomerization in the $S_{1}$ state of hexadecaheptaene
}

\author{
Ronald L. Christensen ${ }^{1}$, Mary Grace I. Galinato ${ }^{2}$, Emily F. Chu ${ }^{2}$, Ritsuko Fujii ${ }^{3}$, Hideki \\ Hashimoto ${ }^{4}$, and Harry A. Frank ${ }^{2}$ \\ 1 Department of Chemistry, Bowdoin College, Brunswick, ME 04011-8466, USA \\ 2 Department of Chemistry, 55 North Eagleville Road, University of Connecticut, Storrs, CT 06269-3060, \\ USA
}

3 Department of Physics, Osaka City University, 3-3-138 Sugimoto, Sumiyoshi-ku, Osaka 558-8585, Japan

4 “Light and Control” PRESTO/JST, 4-1-8 Honcho Kawaguchi, Saitama 332-0012, Japan

\section{Abstract}

The room temperature absorption and emission spectra of the 4-cis and all-trans isomers of 2,4,6,8,10,12,14-hexadecaheptaene are almost identical, exhibiting the characteristic dual emissions $\mathrm{S}_{1} \rightarrow \mathrm{S}_{0}\left(2^{1} \mathrm{~A}_{\mathrm{g}}{ }^{-} \rightarrow 1^{1} \mathrm{~A}_{\mathrm{g}}{ }^{-}\right)$and $\mathrm{S}_{2} \rightarrow \mathrm{S}_{0}\left(1^{1} \mathrm{~B}_{\mathrm{u}}{ }^{+} \rightarrow 1^{1} \mathrm{~A}_{\mathrm{g}}{ }^{-}\right)$noted in previous studies of intermediate length polyenes and carotenoids. The ratio of the $\mathrm{S}_{1} \rightarrow \mathrm{S}_{0}$ and $\mathrm{S}_{2} \rightarrow \mathrm{S}_{0}$ emission yields for the cis isomer increases by a factor of $\sim 15$ upon cooling to $77 \mathrm{~K}$ in $n$-pentadecane. In contrast, for the trans isomer this ratio shows a two-fold decrease with decreasing temperature. These results suggest a low barrier for conversion between the 4-cis and all-trans isomers in the $S_{1}$ state. At $77 \mathrm{~K}$, the cis isomer cannot convert to the more stable all-trans isomer in the $2{ }^{1} \mathrm{~A}_{\mathrm{g}}{ }^{-}$state, resulting in the striking increase in its $S_{1} \rightarrow S_{0}$ fluorescence. These experiments imply that the $S_{1}$ states of longer polyenes have local energy minima, corresponding to a range of conformations and isomers, separated by relatively low ( $2-4 \mathrm{kcal}$ ) barriers. Steady state and time-resolved optical measurements on the $S_{1}$ states in solution thus may sample a distribution of conformers and geometric isomers, even for samples represented by a single, dominant ground state structure. Complex $\mathrm{S}_{1}$ potential energy surfaces may help explain the complicated $S_{2} \rightarrow S_{1}$ relaxation kinetics of many carotenoids. The finding that fluorescence from linear polyenes is so strongly dependent on molecular symmetry requires a reevaluation of the literature on the radiative properties of all-trans polyenes and carotenoids.

\section{INTRODUCTION}

The optical spectroscopy of short, all-trans polyenes reveals an excited $2^{1} \mathrm{~A}_{\mathrm{g}}{ }^{-}$singlet state, into which absorption is forbidden by symmetry, lying at lower energy than the $1^{1} \mathrm{~B}_{\mathrm{u}}{ }^{+}$state responsible for the characteristic strong visible absorption $\left(\mathrm{S}_{0}\left(1^{1} \mathrm{~A}_{\mathrm{g}}^{-}\right) \rightarrow \mathrm{S}_{2}\left(1^{1} \mathrm{~B}_{\mathrm{u}}^{+}\right)\right)$in these $\mathrm{C}_{2 \mathrm{~h}}$ symmetric, linearly-conjugated $\pi$-electron systems. ${ }^{1,2}$ This explains several distinctive aspects of polyene optical spectroscopy, including the systematic differences in the transition energies of the strong absorption and the fluorescence $\left(\mathrm{S}_{1}\left(2^{1} \mathrm{~A}_{\mathrm{g}}{ }^{-}\right) \rightarrow \mathrm{S}_{0}\left(1^{1} \mathrm{~A}_{\mathrm{g}}{ }^{-}\right)\right)$, the anomalously long radiative lifetimes, and the relative insensitivity of the fluorescence spectra to solvent polarizability. ${ }^{2}$ Theoretical analysis by Schulten and Karplus ${ }^{3}$ of short, all-trans polyenes rationalized the low lying $\mathrm{S}_{1}\left(2^{1} \mathrm{~A}_{\mathrm{g}}{ }^{-}\right)$state in terms of extensive configuration interaction between singly and multiply excited singlet configurations with the same symmetry. 
Extensions of this model to longer polyenes and carotenoids predict additional lowlying ${ }^{1} \mathrm{~A}_{\mathrm{g}}{ }^{-}$and ${ }^{1} \mathrm{~B}_{\mathrm{u}}{ }^{-}$excited states ${ }^{4,5}$, but these other states are not easily detected, either in conventional steady-state or time-resolved spectroscopic measurements.

The theoretical descriptions of all-trans polyene excited states have had considerable influence, not only in interpreting the spectroscopy and photophysics of all-trans polyenes, but also in explaining optical measurements on a variety of less symmetric polyenes and carotenoids. For example, Koyama, et al. ${ }^{6,7}$ assigned features in resonance Raman excitation profiles and in the fluorescence spectra of long carotenoids to low-lying $1^{1} \mathrm{~B}_{\mathrm{u}}{ }^{-}$states. This group also used ultrafast optical spectroscopy to detect transient absorption features, again attributed to the $1^{1} \mathrm{~B}_{\mathrm{u}}{ }^{-}$state. 8,9 Cerullo et al. ${ }^{10}$ presented ultrafast spectroscopic evidence for an intermediate singlet state $\left(\mathrm{S}_{\mathrm{X}}\right)$ in several carotenoids, which they postulated facilitates internal conversion between the $\mathrm{S}_{2}\left(1^{1} \mathrm{~B}_{\mathrm{u}}{ }^{+}\right)$and $\mathrm{S}_{1}\left(2^{1} \mathrm{~A}_{\mathrm{g}}{ }^{-}\right)$states. Van Grondelle and coworkers ${ }^{11}$ observed a wavelength dependence of the dynamics of spirilloxanthin that was interpreted in terms of another singlet electronic state, $S^{*}$, thought to be an intermediate in the depopulation of $S_{2}$ $\left(1^{1} \mathrm{~B}_{\mathrm{u}}{ }^{+}\right)$. Fast pump-probe optical techniques were applied to $\beta$-carotene by Larsen, et al. ${ }^{12}$, and the results suggested yet another carotenoid excited state $\left(S^{\ddagger}\right)$ formed directly from $S_{2}$ $\left(1^{1} \mathrm{~B}_{\mathrm{u}}{ }^{+}\right)$. The nature of these states remains uncertain, and recent work has called into question the assignments and suggested that at least some of the spectroscopic observations may be attributed to two-photon processes rather than additional electronic states. ${ }^{13,14}$

Advances in synthetic procedures coupled with improved purification and analytical techniques (HPLC, MS/APCI+, and NMR) have allowed us to revisit the electronic states of simple all-trans dimethyl polyenes and to extend optical experiments to their less symmetric cis counterparts. These studies reveal that the rates of radiative decay from the " $\mathrm{S}_{1}\left(2^{1} \mathrm{~A}_{\mathrm{g}}{ }^{-}\right.$)" states of cis polyenes are significantly larger than radiative decay rates from the $\mathrm{S}_{1}\left(2^{1} \mathrm{~A}_{\mathrm{g}}{ }^{-}\right)$ states of trans polyenes. Our experiments also suggest that trans $\leftrightarrow$ cis conversion readily occurs on the $\mathrm{S}_{1}\left(2^{1} \mathrm{~A}_{\mathrm{g}}{ }^{-}\right)$potential surface. The photochemical formation of $\mathrm{cis}$ isomers from trans ground states requires a reevaluation of previous reports of fluorescence from trans polyenes and carotenoids. Many of these studies were carried out prior to the advent of sophisticated high performance liquid chromatography (HPLC) techniques capable of achieving the high level of sample purity and analysis $2,15-27$ required to identify the source of fluorescence signals. The work presented here provides an alternate model for internal conversion following the excitation of $\mathrm{S}_{2}\left(1^{1} \mathrm{~B}_{\mathrm{u}}{ }^{+}\right)$and suggests that at least some of the electronic states postulated in recent years may be associated with different geometric isomers and/or conformers formed in the $\mathrm{S}_{1}\left(2^{1} \mathrm{~A}_{\mathrm{g}}{ }^{-}\right)$state. Many of the essential features of the simple "three state" scheme, $\mathrm{E}\left(\mathrm{S}_{2}\left(1^{1} \mathrm{~B}_{\mathrm{u}}{ }^{+}\right)\right)>\mathrm{E}\left(\mathrm{S}_{1}\left(2^{1} \mathrm{~A}_{\mathrm{g}}{ }^{-}\right)\right)>\mathrm{E}\left(\mathrm{S}_{0}\left(1^{1} \mathrm{~A}_{\mathrm{g}}{ }^{-}\right)\right)$, are preserved by invoking isomerization and/or conformational change on short time scales in the $\mathrm{S}_{1}\left(2^{1} \mathrm{~A}_{\mathrm{g}}{ }^{-}\right)$ state.

\section{EXPERIMENTAL}

2,4,6,8,10,12,14-hexadecaheptaene was synthesized via a Wittig reaction between 2,4,6,8,10,12-dodecapentaenal and crotyltriphenylphosphonium bromide. The hexadecaheptaene products were isolated using silica gel chromatography and then photolyzed to convert the predominantly cis mixture into the all-trans isomer. $\mathrm{HPLC}\left(\mathrm{C}_{18}\right.$-reverse phase) was used to isolate the cis and trans isomers for spectroscopic analysis. Mass spectrometry (MS/APCI+) and NMR spectroscopy (2D ${ }^{1} \mathrm{H}^{-}{ }^{1} \mathrm{H}$ COSY and NOESY) were used to identify the major product of the Wittig reaction as 4-cis hexadecaheptaene. The main photolysis product is the all-trans isomer, as summarized in the following reaction scheme: 


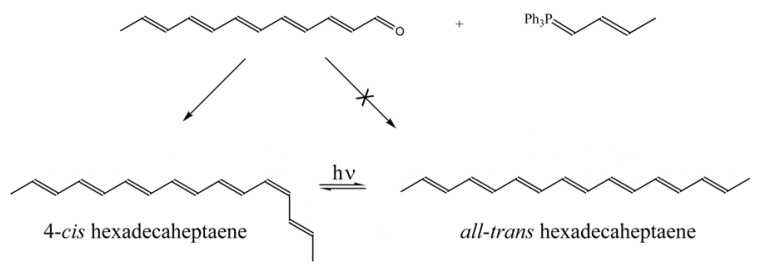

\section{Synthesis of 4-cis hexadecaheptaene}

All-trans 2,4,6,8,10,12-dodecapentaenal was obtained by condensing crotonaldehyde (SigmaAldrich) as described previously. ${ }^{19,28,29} 50 \mathrm{mg}$ of the crotyl ylide (Fluka) was combined with $1 \mathrm{~mL}$ of anhydrous THF in a 5-mL flask. $17 \mathrm{mg}$ of the dodecapentaenal was added and the mixture stirred for 30 minutes. The characteristic polyene absorption (300-400 nm) was used to follow the buildup of the heptaene product. Aqueous $\mathrm{NaOH}$ was added to quench the reaction and the hexadecaheptaene extracted using several aliquots of warm hexane. The crude hexadecaheptaene was purified on silica gel (Silica Gel 60 -EM Reagents) using hexane as a mobile phase.

\section{Photoisomerization and high-performance liquid chromatography (HPLC) of hexadecaheptaene}

Hexadecaheptaene fractions collected from the silica gel column were evaporated and reconstituted in acetonitrile (Fisher Scientific, HPLC-grade) and placed in a $1-\mathrm{cm}$ path length quartz spectrophotometer cell. The sample (Absorbance 1.6) was exposed to $396 \mathrm{~nm}$ light with a $14.7 \mathrm{~nm}$ bandpass using a Jobin-Yvon Horiba Fluorolog-3 fluorescence spectrometer (see below). The illumination was interrupted every 5 minutes to mix the sample and to record the absorption spectrum. The total sample illumination time was 30 minutes. The photolyzed sample was analyzed using a Waters HPLC equipped with a 600S controller, a 616 pump and a 717plus autosampler. A Waters 996 photodiode array detector (PDA) monitored the absorption spectra of the peaks as they eluted from a Nova-Pak reverse-phase C18 column (3.9 $\times 300 \mathrm{~mm}, 60 \AA$ pore size and $4 \mu \mathrm{m}$ particle size of spherical amorphous silica). Acetonitrile was used as the mobile phase, and the system was run in isocratic mode at a flow rate of 0.5 $\mathrm{mL} / \mathrm{min}$.

\section{Characterization of hexadecaheptaene isomers by nuclear magnetic resonance spectroscopy}

The dominant isomer in the unphotolyzed hexadecaheptaene sample (Fig. 1) was isolated by HPLC using a $250 \times 4.6 \mathrm{~mm}$ YMC-Pak $\mathrm{C}_{18}$-A column (5- $\mu \mathrm{m}$ particle size, 12 -nm pore size) and acetonitrile as the mobile phase. $1 \mathrm{D}$ and $2 \mathrm{D}{ }^{1} \mathrm{H}$ NMR spectra, including ${ }^{1} \mathrm{H}-{ }^{1} \mathrm{H}$ COSY and ${ }^{1} \mathrm{H}-{ }^{1} \mathrm{H}$ NOESY spectra, were recorded in chloroform-D at room temperature using a 600 MHz FT NMR spectrometer. Proton signals corresponding to the methyl groups showed distinct chemical shifts, indicating an asymmetric structure. Exploiting the 2D correlation peaks starting from the methyl protons, signals due to the ethylenic protons were assigned based on splittings, coupling constants, and COSY cross peaks between neighboring protons. These assignments are summarized in Figure S1 (Supporting Information). NOE correlations also proved useful in determining the isomeric configuration, e.g., the clear NOE correlation between $3 \mathrm{H}$ and $6 \mathrm{H}$ confirmed that the $4 \mathrm{C}=5 \mathrm{C}$ bond had a cis configuration. Other $\mathrm{NOE}$ correlations confirmed trans geometries for the other double bonds. The isomer thus was assigned unequivocally as 4-cis hexadecaheptaene.

\section{Fluorescence spectroscopy}

Individual HPLC peaks were collected, evaporated, and reconstituted in $n$-pentadecane for fluorescence and fluorescence excitation measurements. Samples were reinjected into the 
HPLC after spectroscopic experiments to verify their isomeric purity after exposure to light. Fluorescence spectra were acquired using a Jobin-Yvon Horiba Fluorolog-3 Model FL3-22 spectrometer equipped with double monochromators with 1200 grooves/mm gratings, a Hamamatsu R928P photomultiplier, and a $450 \mathrm{~W}$ OSRAM XBO xenon arc lamp. The emission was monitored using front-face detection for the low temperature experiments and right-angle detection for the room temperature measurements. A home-built, flowing gaseous $\mathrm{N}_{2}$ quartz cryostat maintained sample temperatures between 77 and $300 \mathrm{~K}$. A gold-chromel thermocouple connected to an Air Products digital temperature controller monitored sample temperatures. For studies of the temperature dependence of fluorescence, purified samples were quickly frozen in liquid nitrogen and then transferred to the flowing $\mathrm{N}_{2}$ cryostat. The temperature was allowed to equilibrate for several minutes before taking each spectrum. Fluorescence spectra were collected systematically both with increasing and with decreasing temperature to understand the effects of photochemistry as well as temperature on the fluorescence intensities of samples that were initially highly-purified isomers. Emission spectra were corrected for the instrument response using a data file generated by a $200 \mathrm{~W}$ standard quartz tungsten-halogen filament lamp with spectral irradiance values traceable to NIST standards. For the display of spectra and the calculation of relative fluorescence yields, the emission spectra were converted to a wavenumber scale and intensities multiplied by $\lambda^{2}$ to give relative emission intensities in photons $/ \mathrm{s} \mathrm{cm}^{-1} \cdot 30$

\section{RESULTS AND DISCUSSION}

Figure 1 shows the HPLC of the hexadecaheptaene sample before and after photoisomerization. One- and two-dimensional NMR spectroscopy $\left({ }^{1} \mathrm{H}-{ }^{1} \mathrm{H}\right.$ COSY and ${ }^{1} \mathrm{H}-{ }^{1} \mathrm{H}$ NOESY) identify the peak eluting at $\sim 8.5 \mathrm{~min}$ as the 4 -cis isomer. This isomer dominates the products of the Wittig reaction, while the all-trans isomer is the major photochemical product. Irradiation of the pure all-trans isomer gives 4-cis hexadecaheptaene as the major photoproduct with the alltrans isomer dominating the photostationary state. The 4-cis and all-trans isomers thus are connected as major, but not exclusive photoisomerization products of each other. Extended irradiation indicates that, in addition to photoisomerization, there are a variety of other photochemical and thermal processes that lead to irreversible degradation of the samples.

Collection of the 4-cis and all-trans fractions from the HPLC allows the comparison of their room temperature absorption and emission spectra in pentadecane (Fig. 2). As noted in previous studies of a wide range of polyenes and carotenoids in solution, $31-33$ the room temperature fluorescence spectra and fluorescence quantum yields of the cis and trans samples are very similar. Following $\mathrm{S}_{0}\left(1^{1} \mathrm{~A}_{\mathrm{g}}{ }^{-}\right) \rightarrow \mathrm{S}_{2}\left({ }^{1} \mathrm{~B}_{\mathrm{u}}{ }^{+}\right)$excitation, both isomers show dual emissions, $\mathrm{S}_{2}\left(1^{1} \mathrm{~B}_{\mathrm{u}}{ }^{+}\right) \rightarrow \mathrm{S}_{0}\left(1^{1} \mathrm{~A}_{\mathrm{g}}{ }^{-}\right)$and $\mathrm{S}_{1}\left(2^{1} \mathrm{~A}_{\mathrm{g}}{ }^{-}\right) \rightarrow \mathrm{S}_{0}\left(1^{1} \mathrm{~A}_{\mathrm{g}}{ }^{-}\right)$, a signature of polyenes and carotenoids with intermediate conjugation lengths $(\mathrm{N} \approx 6-8) .{ }^{17}, 19,20,34,35$ In $n$-pentadecane, the emission and absorption spectra of the heptaenes exhibit sufficient vibronic resolution to allow the identification of the (0-0) bands, providing an accurate measure of the energies of the $S_{1}$ $\left(2^{1} \mathrm{~A}_{\mathrm{g}}{ }^{-}\right)$and $\mathrm{S}_{2}\left(1^{1} \mathrm{~B}_{\mathrm{u}}{ }^{+}\right)$states of these isomers.

The $77 \mathrm{~K}$ fluorescence and fluorescence excitation spectra of 4-cis hexadecaheptaene in $n$ pentadecane are presented in Fig. 3. The high-resolution environment provided by the $n$ pentadecane matrix offer critical advantages in analyzing these spectra. The $n$-alkane mixed crystal selectively incorporates the polyene into a dominant substitutional site, providing welldefined, relatively homogeneous polyene-alkane interactions. This significantly decreases the inhomogeneous spectral broadening inherent to spectra obtained in solutions or lowtemperature glasses. Although only the leading edge of the major 4-cis was collected from the HPLC (Fig. 1), the samples no doubt contain small amounts of other isomers. However, the high-resolution environments provided by the mixed crystals provide enhanced selectivity in exciting and detecting emission from the dominant 4-cis component in fluorescence emission 
and excitation spectra. The use of $n$-alkane host crystals 36,37 has been exploited in several previous optical studies of simple polyenes, ${ }^{18,27,38-40}$ including $2,4,6,8,10,12,14,16$ octadecaoctaene, the longest linear polyene studied using low-temperature, high-resolution mixed-crystal techniques. 40

The spectra of 4-cis hexadecaheptaene presented in Fig. 3 exhibit well-resolved vibronic progressions in the $S_{0}\left(1^{1} A_{g}{ }^{-}\right) \rightarrow S_{2}\left(1^{1} B_{u}{ }^{+}\right)$absorption and the $S_{1}\left(2{ }^{1} A_{g}{ }^{-}\right) \rightarrow S_{0}\left(1^{1} A_{g}{ }^{-}\right)$ emission. As noted in previous studies on model polyenes, $18,27,34,38-40$ these progressions are dominated by combinations of totally symmetric carbon-carbon single and carbon-carbon double bond stretches built on easily identified electronic origins. The low temperature emission spectrum also shows features that can be identified with weak $S_{2}\left(1^{1} B_{u}{ }^{+}\right) \rightarrow S_{0}$ $\left(1^{1} \mathrm{~A}_{\mathrm{g}}{ }^{-}\right)$fluorescence. In addition, the spectra allow the identification of relatively sharp vibronic bands due to the weak $\mathrm{S}_{0}\left(1^{1} \mathrm{~A}_{\mathrm{g}}{ }^{-}\right) \rightarrow \mathrm{S}_{1}\left(2^{1} \mathrm{~A}_{\mathrm{g}}{ }^{-}\right)$transition on the low-energy tail of the strong $\mathrm{S}_{0}\left(1^{1} \mathrm{~A}_{\mathrm{g}}{ }^{-}\right) \rightarrow \mathrm{S}_{2}\left(1^{1} \mathrm{~B}_{\mathrm{u}}{ }^{+}\right)$absorption. This transition is considerably more difficult to detect and identify in the much broader spectra of polyenes and carotenoids in room temperature solutions and low temperature glasses, illustrating another distinct benefit of the $n$-alkane matrices.

In contrast to the emission spectra obtained at room temperature, the $77 \mathrm{~K}$ fluorescence spectra (Fig. 4) of the 4-cis and all-trans isomers in $n$-pentadecane are remarkably different. The $\mathrm{S}_{2}$ $\left(1^{1} \mathrm{~B}_{\mathrm{u}}{ }^{+}\right) \rightarrow \mathrm{S}_{0}\left(1^{1} \mathrm{~A}_{\mathrm{g}}{ }^{-}\right)$emissions exhibit comparable intensities, with the all-trans isomer showing a small red shift in its somewhat better resolved spectrum (Fig. 4B). On the other hand, at $77 \mathrm{~K}$ the $\mathrm{S}_{1}$ (" $\left.2^{1} \mathrm{~A}_{\mathrm{g}}{ }^{-")}\right) \rightarrow \mathrm{S}_{0}$ (" $1{ }^{1} \mathrm{~A}_{\mathrm{g}}{ }^{-"}$ ") emission of the 4-cis isomer is at least 20 times more intense than the $\mathrm{S}_{1}\left(2^{1} \mathrm{~A}_{\mathrm{g}}{ }^{-}\right) \rightarrow \mathrm{S}_{0}\left(1^{1} \mathrm{~A}_{\mathrm{g}}{ }^{-}\right)$emission of the trans isomer (Fig. 4A). The dependence of the 4-cis emission yield on temperature is presented in Fig. 5. In order to compensate for fluctuations in fluorescence intensities in the flowing $\mathrm{N}_{2}$ cryostat, we compare the ratio of integrated fluorescence intensities, $\varphi\left(S_{1} \rightarrow S_{0}\right) / \varphi\left(S_{2} \rightarrow S_{0}\right)$, for the two isomers. For the 4-cis isomer, the factor of $\sim 15$ change in this ratio is almost entirely due to the increase in $\mathrm{S}_{1}\left(2^{1} \mathrm{~A}_{\mathrm{g}}{ }^{-}\right) \rightarrow \mathrm{S}_{0}\left(1^{1} \mathrm{~A}_{\mathrm{g}}{ }^{-}\right)$fluorescence upon cooling to $77 \mathrm{~K}$. The corresponding ratio in the all-trans isomer shows a two-fold decrease when the sample is cooled. It is important to note

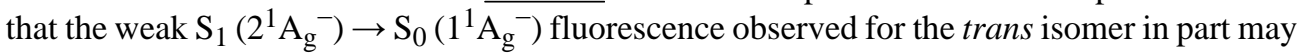
be due to the photochemical production of highly fluorescent 4-cis and other cis impurities. The data indicated in Fig. 4 and 5 thus may underestimate the true difference between the low temperature $\mathrm{S}_{1}\left(2^{1} \mathrm{~A}_{\mathrm{g}}\right) \rightarrow \mathrm{S}_{0}\left(1^{1} \mathrm{~A}_{\mathrm{g}}{ }^{-}\right)$fluorescence yields of the pure 4-cis and pure all-trans isomers.

The $n$-alkane host offers a significant advantage for detecting the differences between $\mathrm{S}_{1}$ $\left(2^{1} \mathrm{~A}_{\mathrm{g}}{ }^{-}\right) \rightarrow \mathrm{S}_{0}\left(1^{1} \mathrm{~A}_{\mathrm{g}}{ }^{-}\right)$fluorescence yields in the two isomers at low temperature. The $n$ pentadecane mixed crystal preserves the planar, symmetric $\left(\mathrm{C}_{2 \mathrm{~h}}\right)$ geometry of the all-trans heptaene, leading to relatively slow radiative decay for the symmetry-forbidden, $\mathrm{S}_{1}\left(2^{1} \mathrm{~A}_{\mathrm{g}}{ }^{-}\right)$ $\rightarrow \mathrm{S}_{0}\left(1^{1} \mathrm{~A}_{\mathrm{g}}{ }^{-}\right)$transition. Furthermore, the $n$-pentadecane environment provides sufficient optical resolution to differentiate between emissions due to the 4-cis and the all-trans species (Fig. 4). This is particularly important in distinguishing the weak emissions from the trans species from those of much more strongly emitting cis photochemical products. The differences between the fluorescence intensities of cis and trans samples are considerably less distinctive in the random environments provided by low temperature glasses (data not shown), which produce a distribution of distorted trans polyenes, many of which do not have $\mathrm{C}_{2 \mathrm{~h}}$ symmetry.

The current work recalls a previous controversy regarding the origin of the fluorescence signals (cis impurities versus the dominant trans species) in samples of cold, isolated octatetraene, the most highly studied and best-understood linear polyene. Buma et al. ${ }^{41}$ assigned the isolated molecule, $\mathrm{S}_{0}\left(1^{1} \mathrm{~A}_{\mathrm{g}}{ }^{-}\right) \rightarrow \mathrm{S}_{1}\left(2^{1} \mathrm{~A}_{\mathrm{g}}{ }^{-}\right)$fluorescence excitation spectrum (obtained in a resonanceenhanced multiphoton ionization (REMPI) measurement) to a mono-cis isomer, arguing that 
the trans species would have insufficient oscillator strength for fluorescence detection. A subsequent study by Petek, et al., ${ }^{42}$ on the high-resolution one- and two-photon fluorescence excitation spectra of octatetraene in supersonic jets, demonstrated that the oscillator strengths for the $\mathrm{S}_{0}\left(1^{1} \mathrm{~A}_{\mathrm{g}}{ }^{-}\right) \leftrightarrow \mathrm{S}_{1}\left(2^{1} \mathrm{~A}_{\mathrm{g}}{ }^{-}\right)$transitions in the trans isomer (induced by Herzberg-Teller vibronic coupling via low frequency $b_{u}$ promoting modes) were comparable to the corresponding oscillator strengths for $c i s$ isomers. This results in fluorescence intensities from the cis and trans isomers of isolated octatetraene that mirror their ground state abundances, i.e., the fluorescence spectra can be identified with the dominant trans isomer in typical samples. The identification of all-trans octatetraene as the dominant emitting species was confirmed by analysis of the rotationally resolved $\mathrm{S}_{0}\left(1^{1} \mathrm{~A}_{\mathrm{g}}{ }^{-}\right) \rightarrow \mathrm{S}_{1}\left(2^{1} \mathrm{~A}_{\mathrm{g}}{ }^{-}\right)$fluorescence excitation spectrum by Pfanstiel, et al. ${ }^{43}$ These experiments established that the absorbing and emitting states both had all-trans, planar geometries and also demonstrated that the $\mathrm{S}_{0}$ $\left(1^{1} \mathrm{~A}_{\mathrm{g}}{ }^{-}\right) \leftrightarrow \mathrm{S}_{1}\left(2^{1} \mathrm{~A}_{\mathrm{g}}{ }^{-}\right)$electronic transitions gain their intensities via vibronic coupling with the $\mathrm{S}_{2}\left(1^{1} \mathrm{~B}_{\mathrm{u}}{ }^{+}\right)$state.

The work presented here shows that, in contrast to octatetraene, the 4-cis isomer of hexadecaheptaene has a much higher $\mathrm{S}_{0} \leftrightarrow \mathrm{S}_{1}$ oscillator strength than its all-trans counterpart. Under conditions where the trans isomer can be described by $\mathrm{C}_{2 \mathrm{~h}}$ symmetry, e.g., in lowtemperature $n$-pentadecane, the vibronically induced, $\mathrm{S}_{1}\left(2^{1} \mathrm{~A}_{\mathrm{g}}{ }^{-}\right) \rightarrow \mathrm{S}_{0}\left(1^{1} \mathrm{Ag}_{\mathrm{g}}^{-}\right)$radiative decay is relatively slow, and the fluorescence can be dominated by heptaenes with distorted, s-cis or cis conformations, even though these species may be present in relatively low concentrations. The ability of cis isomers to dominate the fluorescence systematically increases with increasing conjugation length (manuscript in preparation).

The striking difference in temperature dependence of the fluorescence from the two isomers (Fig. 5) leads to the simple, qualitative model presented in Fig. 6 . The thermodynamically favored all-trans isomer is connected to the 4-cis isomer by a relatively low energy barrier in the $\mathrm{S}_{1}\left(2^{1} \mathrm{~A}_{\mathrm{g}}{ }^{-}\right)$state. Our preliminary photochemical studies (Fig. 1) indicate that additional cis isomers also are accessible on the $\mathrm{S}_{1}\left(2^{1} \mathrm{~A}_{\mathrm{g}}{ }^{-}\right)$potential surface. Theoretical considerations also implicate s-cis, as well as distorted trans isomers on a complicated, multi-dimensional potential surface with many local minima, connected by relatively low barriers. However, since the 4-cis and all-trans isomers are the two major products of all-trans and 4-cis

photoisomerization, we only consider these isomers in a much-simplified model (Fig. 6). The lower energy, all-trans isomer dominates the photostationary state, a common theme in polyene and carotenoid photochemistry. ${ }^{44}$ Our model (Fig. 6) does not imply a mechanism for the isomerization; a detailed microscopic understanding of the chemistry would require additional experimental and theoretical work.

For the model presented in Figure 6, the $\mathrm{S}_{1}\left(2^{1} \mathrm{~A}_{\mathrm{g}}{ }^{-}\right) \rightarrow \mathrm{S}_{0}\left(1^{1} \mathrm{~A}_{\mathrm{g}}{ }^{-}\right)$fluorescence quantum yield for the 4-cis isomer can be approximated by:

$$
\varphi(\mathrm{T})=\mathrm{k}_{\mathrm{r}} /\left(\mathrm{k}_{\mathrm{r}}+\mathrm{k}_{\mathrm{nr}}+\mathrm{Ae}^{-\mathrm{E}_{\mathrm{a}} / \mathrm{RT}}\right)
$$

We assume that the radiative and nonradiative decay rates are independent of temperature and that the 4-cis $\rightarrow$ trans isomerization proceeds over an activation barrier $\mathrm{E}_{\mathrm{a}}$. We also assume that the $\mathrm{S}_{2}\left(1^{1} \mathrm{~B}_{\mathrm{u}}{ }^{+}\right) \rightarrow \mathrm{S}_{0}\left(1^{1} \mathrm{~A}_{\mathrm{g}}{ }^{-}\right)$fluorescence yield does not depend on temperature in fitting the data presented in Figure 5 to Equation 1. The resulting four-parameter fit (see Fig. 5) yields an activation energy of $4.3 \pm 1.4 \mathrm{kcal}$ and a pre-exponential factor (A) of $10^{10}-10^{12} \mathrm{~s}^{-1}$. The fit is poorly determined, e.g., the parameters for the activation energy and pre-exponential factor are highly correlated with large uncertainties. The fits are heavily influenced by the high temperature data points, which include the melting of the $n$-pentadecane crystal at $10{ }^{\circ} \mathrm{C}$. In addition to the least squares analysis, we also have employed simulations to explore the parameter space and to better understand the data and the model (Equation 1). The determination of relative fluorescence yields as a function of temperature on samples 
undergoing photoisomerization is particularly challenging, especially for weakly emitting species. In spite of the limitations both of the model and the data, the parameters extracted from Equation 1 are consistent with thermally activated, 4-cis $\rightarrow$ trans isomerization in the $\mathrm{S}_{1}$ state.

An identical model was postulated to account for the temperature dependence of $S_{1}$ lifetimes of $c i s$ and trans octatetraenes in different $n$-alkane solvents. ${ }^{45}$ The temperature-dependences of the octatetraene $S_{1}$ lifetimes between $77 \mathrm{~K}$ and room temperature are comparable to the data presented in Fig. 5 and also were interpreted in terms of a thermally activated cis $\leftrightarrows$ trans isomerization in $S_{1}$ with pre-exponential factors of $10^{10}-10^{12} \mathrm{~s}^{-1}$ and activation energies of a few $\mathrm{kcal} / \mathrm{mol} .{ }^{45,46}$ For example, analysis of lifetime data indicated an $S_{1}$ barrier of $1.1 \mathrm{kcal}$ for the isomerization of cis, trans-1,3,5,7-octatetraene to the all-trans isomer ${ }^{47}$ and $2.5 \mathrm{kcal}$ for the reverse process. ${ }^{45}$ The connections between the temperature dependence of the fluorescence lifetimes of octatetraene isomers and the fluorescent quantum yield data presented in Figure 5 with excited state isomerizations are buttressed by the temperature dependence of the photochemical quantum yields for trans $\rightarrow$ cis isomerizations in all-trans retinal. These studies indicate activation barriers of 1.6-3.2 kcal, depending on the solvent and the cis isomer formed. ${ }^{48}$ It also is important to note that the barriers to $\mathrm{C}=\mathrm{C}$ isomerization in the $\mathrm{S}_{1}$ state are comparable to those for isomerization of carbon-carbon single bonds in polyene ground states. 46,49 This reflects the significant rearrangement of the $\mathrm{C}-\mathrm{C}$ and $\mathrm{C}=\mathrm{C} \pi$-bond orders in going from the ground to the $S_{1}$ state. 50

The low thermal barrier associated with the $>20$-fold increase in the $\mathrm{S}_{1}\left(2^{1} \mathrm{~A}_{\mathrm{g}}{ }^{-}\right) \rightarrow \mathrm{S}_{0}$ $\left(1^{1} \mathrm{~A}_{\mathrm{g}}{ }^{-}\right)$fluorescence intensity with decreasing temperature cannot be explained by isomerization on the ground state $\mathrm{S}_{0}\left(1^{1} \mathrm{~A}_{\mathrm{g}}{ }^{-}\right)$potential energy surface. It also is not consistent with isomerization in the short-lived $\mathrm{S}_{2}$ state $\left(1^{1} \mathrm{~B}_{\mathrm{u}}{ }^{+}\right)$. If that were the case, we would expect a substantial change with temperature in the quantum yield of $\mathrm{S}_{2}\left(1^{1} \mathrm{~B}_{\mathrm{u}}{ }^{+}\right) \rightarrow \mathrm{S}_{0}\left(1^{1} \mathrm{~A}_{\mathrm{g}}{ }^{-}\right)$ emission, which is not observed. The increase in the ratio, $\varphi\left(\mathrm{S}_{1} \rightarrow \mathrm{S}_{0}\right) / \varphi\left(\mathrm{S}_{2} \rightarrow \mathrm{S}_{0}\right)$, for the 4cis isomer, thus is almost entirely due to the increase in $\mathrm{S}_{1}\left(2^{1} \mathrm{~A}_{\mathrm{g}}{ }^{-}\right) \rightarrow \mathrm{S}_{0}\left(1^{1} \mathrm{~A}_{\mathrm{g}}{ }^{-}\right)$fluorescence upon cooling. Furthermore, in solutions, trans $\leftrightarrow$ cis isomerization in $\mathrm{S}_{1}\left(2^{1} \mathrm{~A}_{\mathrm{g}}{ }^{-}\right)$must compete with rapid nonradiative processes, which tend to dominate the excited state decay of longer polyenes and carotenoids. ${ }^{20}$

The all-trans heptaene $\mathrm{S}_{1} \rightarrow \mathrm{S}_{0}$ fluorescence yield shows a two-fold increase with temperature (Figure 5), suggesting that it crosses over a barrier in $S_{1}$ to a more fluorescent species. It is tempting to ascribe this weak emission as due to adiabatic conversion to 4-cis heptaene in our simple model. This would account for the rather similar room temperature fluorescence spectra of the all-trans and 4-cis species (Fig. 2) and the fact that the 4-cis isomer is the dominant photoisomerization product of all-trans-hexadecaheptaene. However, the very small fluorescence yields of the all-trans isomer at all temperatures make it difficult to identify its fluorescence with a particular asymmetric species. Adiabatic pathways to other cis isomers, distorted trans conformers, as well as isomerization upon relaxation to the ground state all could induce fluorescence in samples of pure, $\mathrm{C}_{2 \mathrm{~h}}$, all-trans hexadecaheptaene. It thus is much easier to make the case for 4-cis $\rightarrow$ all-trans adiabatic $S_{1}$ conversion than for the reverse process.

The potential energy diagram presented in Fig. 6 is similar to that proposed by de Weerd et al. 51 based on subpicosecond dynamics studies of all-trans $\beta$-carotene. However, those authors suggested that $\beta$-carotene became distorted in the $\mathrm{S}_{2}\left(1^{1} \mathrm{~B}_{\mathrm{u}}{ }^{+}\right)$state and relaxed back to the alltrans configuration upon decaying to $\mathrm{S}_{1}\left(2^{1} \mathrm{~A}_{\mathrm{g}}{ }^{-}\right)$. This is not the case for hexadecaheptaene, and other investigators have argued that conformational twisting occurs in $\mathrm{S}_{1}\left(2^{1} \mathrm{~A}_{\mathrm{g}}{ }^{-}\right)$in $\beta$ carotene $^{52}$ and other carotenoids. ${ }^{52-54}$ 
The relatively large oscillator strength for the $\mathrm{S}_{0}\left(1^{1} \mathrm{~A}_{\mathrm{g}}{ }^{-}\right) \leftrightarrow \mathrm{S}_{1}\left(2^{1} \mathrm{~A}_{\mathrm{g}}{ }^{-}\right)$transition in 4-cis hexadecaheptaene is confirmed by our ability to detect the $\mathrm{S}_{0}\left(1^{1} \mathrm{~A}_{\mathrm{g}}{ }^{-}\right) \rightarrow \mathrm{S}_{1}\left(2^{1} \mathrm{~A}_{\mathrm{g}}{ }^{-}\right)$transition in the high-resolution fluorescence excitation spectrum (Fig. 7). The $\sim 4700 \mathrm{~cm}^{-1}$ gap between the $1^{1} \mathrm{~B}_{\mathrm{u}}{ }^{+}$and $2^{1} \mathrm{~A}_{\mathrm{g}}{ }^{-}$states provides a wide window for observing the vibronic development of the $2^{1} \mathrm{Ag}_{\mathrm{g}}{ }^{-}$vibronic states built on the electronic origin (0-0) at $512 \mathrm{~nm}$. Note also the coincidence between the $512 \mathrm{~nm}(0-0)$ bands in the $\mathrm{S}_{0}\left(1^{1} \mathrm{~A}_{\mathrm{g}}{ }^{-}\right) \rightarrow \mathrm{S}_{1}\left(2^{1} \mathrm{~A}_{\mathrm{g}}{ }^{-}\right)$excitation spectrum (Fig. 7) and the $\mathrm{S}_{1}\left(2^{1} \mathrm{~A}_{\mathrm{g}}{ }^{-}\right) \rightarrow \mathrm{S}_{0}\left(1^{1} \mathrm{~A}_{\mathrm{g}}{ }^{-}\right)$emission spectrum (Fig. 3). The overlap between electronic origins and the relatively strong $S_{0} \rightarrow S_{1}$ absorption indicate a symmetryallowed electronic transition, as expected for the 4-cis isomer. A large $S_{0} \leftrightarrow S_{1}$ oscillator strength explains the considerable enhancement in the 4-cis $\mathrm{S}_{1} \rightarrow \mathrm{S}_{0}$ fluorescence yield compared with that associated with the symmetry forbidden transition of the all-trans isomer. The excitation spectra for both the $\mathrm{S}_{0} \rightarrow \mathrm{S}_{1}$ and $\mathrm{S}_{0} \rightarrow \mathrm{S}_{2}$ absorptions show the classic pattern of vibronic intensities built on combinations of carbon-carbon single and double bond symmetric stretching modes. The vibronic spectrum shown in Fig. 7 previously was analyzed by Simpson et al. and assigned to the all-trans isomer. ${ }^{18}$ However, our current work shows that the vibronic states observed are due to 4-cis hexadecaheptaene.

\section{CONCLUSIONS}

The results presented here require a reinterpretation of fluorescence experiments previously carried out on all-trans isomers of longer polyenes and of related carotenoids. Several previous studies of longer linear polyenes $(\mathrm{N}>4)$ have assigned $\mathrm{S}_{1} \rightarrow \mathrm{S}_{0}$ fluorescence signals to alltrans isomers. Examples include the high-resolution work of Simpson et al. on hexadecaheptaene ${ }^{18}$ and of Kohler et al. on octadecaoctaene. ${ }^{40}$ The excitation and fluorescence spectra were assigned to all-trans species, but our work indicates that the $S_{1} \rightarrow$ $\mathrm{S}_{0}$ emission spectra of these longer polyenes most likely are due to $c i s$ isomers, present as impurities or formed as photochemical products in the $S_{1}$ state. This is a significant finding, given that existing theoretical work (almost all on simple all-trans polyenes) ${ }^{4,5,55,56}$ has been compared with experimental work on what now must be assigned to $c i s$ species. Rapid isomerization in $\mathrm{S}_{1}\left(2^{1} \mathrm{~A}_{\mathrm{g}}{ }^{-}\right)$explains the typically small differences between the $\mathrm{S}_{1}\left(2^{1} \mathrm{Ag}_{\mathrm{g}}{ }^{-}\right)$ $\rightarrow \mathrm{S}_{0}\left(1^{1} \mathrm{~A}_{\mathrm{g}}{ }^{-}\right)$emission spectra and quantum yields of cis and trans systems in room temperature solutions. The almost negligible $\mathrm{S}_{1}\left(2^{1} \mathrm{~A}_{\mathrm{g}}{ }^{-}\right) \rightarrow \mathrm{S}_{0}\left(1^{1} \mathrm{~A}_{\mathrm{g}}{ }^{-}\right)$fluorescence yields from $\mathrm{C}_{2 \mathrm{~h}}$, trans species and the relatively low resolution of solution and glass spectra prohibit a ready distinction between emissions due to trans isomers from those due to cis impurities or from trans molecules with conformational distortions that relax the rigorous selection rules. We thus conclude that, except for the very detailed studies of all-trans octatetraene, previous reports of $S_{1}$ emissions from all-trans, $C_{2 h}$ polyenes and carotenoids most likely are due to less symmetric species. These species may be present as ground state impurities, including photochemical products, or formed in the $\mathrm{S}_{1}\left(2^{1} \mathrm{~A}_{\mathrm{g}}{ }^{-}\right)$state following the excitation of alltrans polyenes.

Our results suggest that steady-state fluorescence experiments and time-resolved measurements, e.g., $S_{1} \rightarrow S_{N}$ transient absorption experiments, detect different distributions of $\mathrm{S}_{1}\left(2^{1} \mathrm{~A}_{\mathrm{g}}{ }^{-}\right)$conformers and geometric isomers, even for samples with a single, all-trans, ground state structure. For example, the elegant $\mathrm{S}_{1}\left(2^{1} \mathrm{~A}_{\mathrm{g}}{ }^{-}\right) \rightarrow \mathrm{S}_{2}\left(1^{1} \mathrm{~B}_{\mathrm{u}}{ }^{+}\right)$absorption experiments of Polívka et al. ${ }^{57}$ on several all-trans carotenoids, including spheroidene, zeaxanthin and violaxanthin, were compared with the transition energies for the strongly allowed $\mathrm{S}_{0}\left(1^{1} \mathrm{~A}_{\mathrm{g}}{ }^{-}\right) \rightarrow \mathrm{S}_{2}\left(1^{1} \mathrm{~B}_{\mathrm{u}}{ }^{+}\right)$absorptions. The energy difference in the electronic origins $\left((0-0)\right.$ bands) of these two symmetry-allowed transitions yields the $\mathrm{S}_{1}\left(2^{1} \mathrm{~A}_{\mathrm{g}}{ }^{-}\right)$energy.

However, $S_{1}$ electronic energies obtained in this manner were found to be consistently 500 $1000 \mathrm{~cm}^{-1}$ lower than those from the fluorescence measurements. This is in accord with the model presented in Fig. 6. Fluorescence from these samples is from higher energy, distorted trans and/or cis carotenoids with $\mathrm{S}_{1} \leftrightarrow \mathrm{S}_{0}$ oscillator strengths and radiative decay rates that 
are sufficiently large to compete with the rapid ( 10 ps) nonradiative decays in these molecules. It should be noted that Polívka et al. ${ }^{58,59}$ suggested a similar model, though did not invoke isomerization, to explain the discrepancies between their results and the $\mathrm{S}_{1}\left(2^{1} \mathrm{~A}_{\mathrm{g}}{ }^{-}\right)$energies estimated by fluorescence techniques. Another conclusion from the current studies is that, at least for longer polyenes and related carotenoids, "forbidden" electronic transitions for molecules with $\mathrm{C}_{2 \mathrm{~h}}$ symmetries will be very difficult to detect. Optical techniques that exploit the selection rules for allowed transitions, e.g., $\mathrm{S}_{1}\left(2^{1} \mathrm{~A}_{\mathrm{g}}{ }^{-}\right) \rightarrow \mathrm{S}_{2}\left(1^{1} \mathrm{~B}_{\mathrm{u}}{ }^{+}\right)$absorption, thus have obvious advantages in accurately locating the excited electronic energy levels of trans polyenes and carotenoids.

The low energy barriers to isomerization and conformational distortion in $\mathrm{S}_{1}\left(2^{1} \mathrm{~A}_{\mathrm{g}}{ }^{-}\right)$are consistent with significant rearrangements of the ground state $\mathrm{C}-\mathrm{C}$ and $\mathrm{C}=\mathrm{C}$ bond orders relative to the changes in $\mathrm{S}_{2}\left(1^{1} \mathrm{~B}_{\mathrm{u}}{ }^{+}\right)$and other low-energy excited states. ${ }^{3-5}$ This transposition of $\pi$-bond orders is a hallmark of polyene electronic structure and explains the unique ability of $S_{1}$ states to promote rapid isomerization. Low barriers to isomerization and conformational change also may account for the complex kinetics of $\mathrm{S}_{2}\left(1^{1} \mathrm{~B}_{\mathrm{u}}{ }^{+}\right) \rightarrow \mathrm{S}_{1}\left(2^{1} \mathrm{~A}_{\mathrm{g}}{ }^{-}\right)$nonradiative decay in carotenoids. As mentioned previously, Cerullo, et al. ${ }^{10}$ postulate the existence of an excited electronic state $\left(S_{\mathrm{x}}\right)$ between $\mathrm{S}_{2}\left(1^{1} \mathrm{~B}_{\mathrm{u}}{ }^{+}\right)$and $\mathrm{S}_{1}\left(2^{1} \mathrm{~A}_{\mathrm{g}}{ }^{-}\right)$that decays on a $\sim 100 \mathrm{fs}$ time scale, and van Grondelle et al. ${ }^{11}$ hypothesize that additional short-lived electronic singlet states ( $\mathrm{S}^{*}$ and $\mathrm{S}^{\ddagger}$ ) provide alternate routes for internal conversion from $\mathrm{S}_{2}$. The current work strongly suggests that at least some of these proposed singlet electronic states instead may be manifestations of nonradiative decay on complicated $\mathrm{S}_{1}\left(2^{1} \mathrm{Ag}_{\mathrm{g}}^{-}\right)$potential surfaces. These surfaces provide multiple pathways for the zero-point level of $S_{2}\left(1^{1} B_{u}{ }^{+}\right)$to change its geometry to arrive at vibrationally relaxed, thermally equilibrated $\mathrm{S}_{1}\left(2^{1} \mathrm{~A}_{\mathrm{g}}{ }^{-}\right)$.

\section{Supplementary Material}

Refer to Web version on PubMed Central for supplementary material.

\section{Acknowledgements}

We thank Tomáš Polívka and Robert Birge for fruitful discussions. RLC has been supported by the Bowdoin College Kenan and Porter Fellowship Programs and acknowledges funding from NSF-ROA (MCB-0314380 to HAF) and the Petroleum Research Fund, administered by the American Chemical Society. RLC also was supported in this work while serving at the National Science Foundation. This research is supported in the laboratory of HAF by the National Institutes of Health (GM-30353) and the University of Connecticut Research Foundation. HH and RF acknowledge grants-in-aid (\# 17204026 and 17654083) from the Japanese Ministry of Education, Culture, Sports, Science and Technology. HH and RF also acknowledge financial support from the Strategic International Cooperative Program of the Japan Science and Technology Agency. We also acknowledge the helpful comments of one of the referees in clarifying our understanding of the role of adiabatic photoisomerization in hexadecaheptaene.

\section{References}

1. Hudson B, Kohler B. Ann Rev Phys Chem 1974;25:437-460.

2. Hudson, BS.; Kohler, BE.; Schulten, K. Linear polyene electronic structure and potential surfaces. In: Lim, ED., editor. Excited States. 6. Academic Press; New York: 1982. p. 1-95.

3. Schulten K, Karplus M. Chem Phys Lett 1972;14:305-309.

4. Tavan P, Schulten K. J Chem Phys 1986;85:6602-6609.

5. Tavan P, Schulten K. Phys Rev B: Condens Matter 1987;36:4337-4358. [PubMed: 9943414]

6. Sashima T, Koyama Y, Yamada T, Hashimoto H. J Phys Chem B 2000;104:5011-5019.

7. Fujii R, Onaka K, Nagae H, Koyama Y, Watanabe Y. J Luminescence 2001;92:213-222.

8. Zhang JP, Inaba T, Watanabe Y, Koyama Y. Chem Phys Lett 2000;332:351-358.

9. Koyama Y, Rondonuwu FS, Fujii R, Watanabe Y. Biopolymers 2004;74:2-18. [PubMed: 15137086]

10. Cerullo G, Polli D, Lanzani G, De Silvestri S, Hashimoto H, Cogdell RJ. Science 2002;298:23952398. [PubMed: 12493917] 
11. Gradinaru CC, Kennis JTM, Papagiannakis E, van Stokkum IHM, Cogdell RJ, Fleming GR, Niederman RA, van Grondelle R. Proc Natl Acad Sci USA 2001;98:2364-2369. [PubMed: 11226245]

12. Larsen DS, Papagiannakis E, van Stokkum IHM, Vengris M, Kennis JTM, van Grondelle R. Chem Phys Lett 2003;381:733-742.

13. Kukura P, McCamant DW, Mathies RA. J Phys Chem A 2004:108.

14. Kosumi D, Komukai M, Hashimoto H, Yoshizawa M. Phys Rev Lett 2005;95:213601-213604. [PubMed: 16384139]

15. Kohler BE, Terpougov V. J Chem Phys 1998;108:9586-9593.

16. Christensen RL, Kohler BE. J Phys Chem 1976;80:2197-2200.

17. Christensen RL, Barney EA, Broene RD, Galinato MGI, Frank HA. Arch Biochem Biophys 2004;430:30-36. [PubMed: 15325909]

18. Simpson JH, McLaughlin L, Smith DS, Christensen RL. J Chem Phys 1987;87:3360-3365.

19. Snyder R, Arvidson E, Foote C, Harrigan L, Christensen RL. J Am Chem Soc 1985;107:4117-4122.

20. Frank HA, Josue JS, Bautista JA, van der Hoef I, Jansen FJ, Lugtenburg J, Wiederrecht G, Christensen RL. J Phys Chem B 2002;106:2083-2092.

21. Shima S, Ilagan RP, Gillespie N, Sommer BJ, Hiller RG, Sharples FP, Frank HA, Birge RR. J Phys Chem A 2003;107:8052-8066.

22. Fujii R, Onaka K, Kuki M, Koyama Y, Watanabe Y. Chem Phys Lett 1998;288:847-853.

23. Josue JS, Frank HA. J Phys Chem A 2002;106:4815-4824.

24. Frank HA, Bautista JA, Josue JS, Young AJ. Biochemistry 2000;39:2831-2837. [PubMed: 10715102]

25. Onaka K, Fujii R, Nagae H, Kuki M, Koyama Y, Watanabe Y. Chem Phys Lett 1999;315:75-81.

26. Andersson PO, Bachilo SM, Chen RL, Gillbro T. J Phys Chem 1995;99:16199-16209.

27. Auerbach RA, Christensen RL, Granville MF, Kohler BE. J Chem Phys 1981;74:4-9.

28. D’Amico KL, Manos C, Christensen RL. J Am Chem Soc 1980;102:1777-1782.

29. Palmer B, Jumper B, Hagan W, Baum JC, Christensen RL. J Am Chem Soc 1982;104:6907-6913.

30. Lakowicz, JR. Principles of Fluorescence Spectroscopy. 2. Kluwer Academic, Plenum Publishers; New York: 1999.

31. Bautista JA, Chynwat V, Cua A, Jansen FJ, Lugtenburg J, Gosztola D, Wasielewski MR, Frank HA. Photosyn Res 1998;55:49-65.

32. Andersson PA, Takaichi S, Cogdell RJ, Gillbro T. Photochem Photobiol 2001;74:549-557. [PubMed: 11683034]

33. Frank HA, Desamero RZB, Chynwat V, Gebhard R, van der Hoef I, Jansen FJ, Lugtenburg J, Gosztola D, Wasielewski MR. J Phys Chem A 1997;101:149-157.

34. Christensen, RL. The electronic states of carotenoids. In: Frank, HA.; Young, AJ.; Britton, G.; Cogdell, RJ., editors. The Photochemistry of Carotenoids. 8. Kluwer Academic Publishers; Dordrecht: 1999. p. 137-159.

35. DeCoster B, Christensen RL, Gebhard R, Lugtenburg J, Farhoosh R, Frank HA. Biochim Biophys Acta 1992;1102:107-114. [PubMed: 1510992]

36. Shpol'skii EV. Sov Phys Usp 1962;5:522-531.

37. Shpol'skii EV. Sov Phys Usp 1963;6:411-427.

38. Christensen RL, Kohler BE. J Chem Phys 1975;63:1837-1846.

39. Andrews JR, Hudson BS. J Chem Phys 1978;68:4587-4594.

40. Kohler BE, Spangler C, Westerfield C. J Chem Phys 1988;89:5422-5428.

41. Buma WJ, Kohler BE, Shaler TA. J Chem Phys 1992;96:399-407.

42. Petek H, Bell AJ, Choi YS, Yoshihara K, Tounge BA, Christensen RL. J Chem Phys 1993;98:37773794.

43. Pfanstiel JF, Pratt DW, Tounge BA, Christensen RL. J Phys Chem A 1999;103:2337-2347.

44. Zechmeister, L. Cis-trans isomeric carotenoids, vitamin A, and arylpolyenes. Academic Press; New York: 1962.

45. Kohler BE, Mitra P, West P. J Chem Phys 1986;85:4436-4440. 
46. Kohler BE. Chem Rev 1993;93:41-54.

47. Ackerman JR, Kohler BE. J Am Chem Soc 1984;106:3681-3682.

48. Waddell WH, Chihara K. J Am Chem Soc 1981;103:7389-7390.

49. Ackerman JR, Kohler BE. J Chem Phys 1984;80:45-50.

50. Schulten K, Ohmine I, Karplus M. J Chem Phys 1976;64:4422-4441.

51. de Weerd FL, van Stokkum IHM, van Grondelle R. Chem Phys Lett 2002;354:38-43.

52. Kosumi D, Yanagi K, Nishio T, Hashimoto HMY. Chem Phys Lett 2005;408:89-95.

53. Billsten HH, Pan J, Sinha S, Pascher T, Sundström V, Polívka T. J Phys Chem A 2005;109:68526859. [PubMed: 16834041]

54. Pendon ZD, Gibson GN, van der Hoef I, Lugtenburg J, Frank HA. J Phys Chem B 2005;109:2117221179. [PubMed: 16853743]

55. Tavan P, Schulten K. J Chem Phys 1979;70:5407-5413.

56. Head-Gordon M, Rico RJ, Oumi M, Lee TJ. Chem Phys Lett 1994;219:21-29.

57. Polívka T, Herek JL, Zigmantas D, Akerlund HE, Sundström V. Proc Nat Acad Sci USA 1999;96:4914-4917. [PubMed: 10220393]

58. Polívka T, Zigmantas D, Frank HA, Bautista JA, Herek JL, Koyama Y, Fujii R, Sundström V. J Phys Chem B 2001;105:1072-1080.

59. Polívka T, Sundström V. Chem Rev 2004;104:2021-2071. [PubMed: 15080720] 


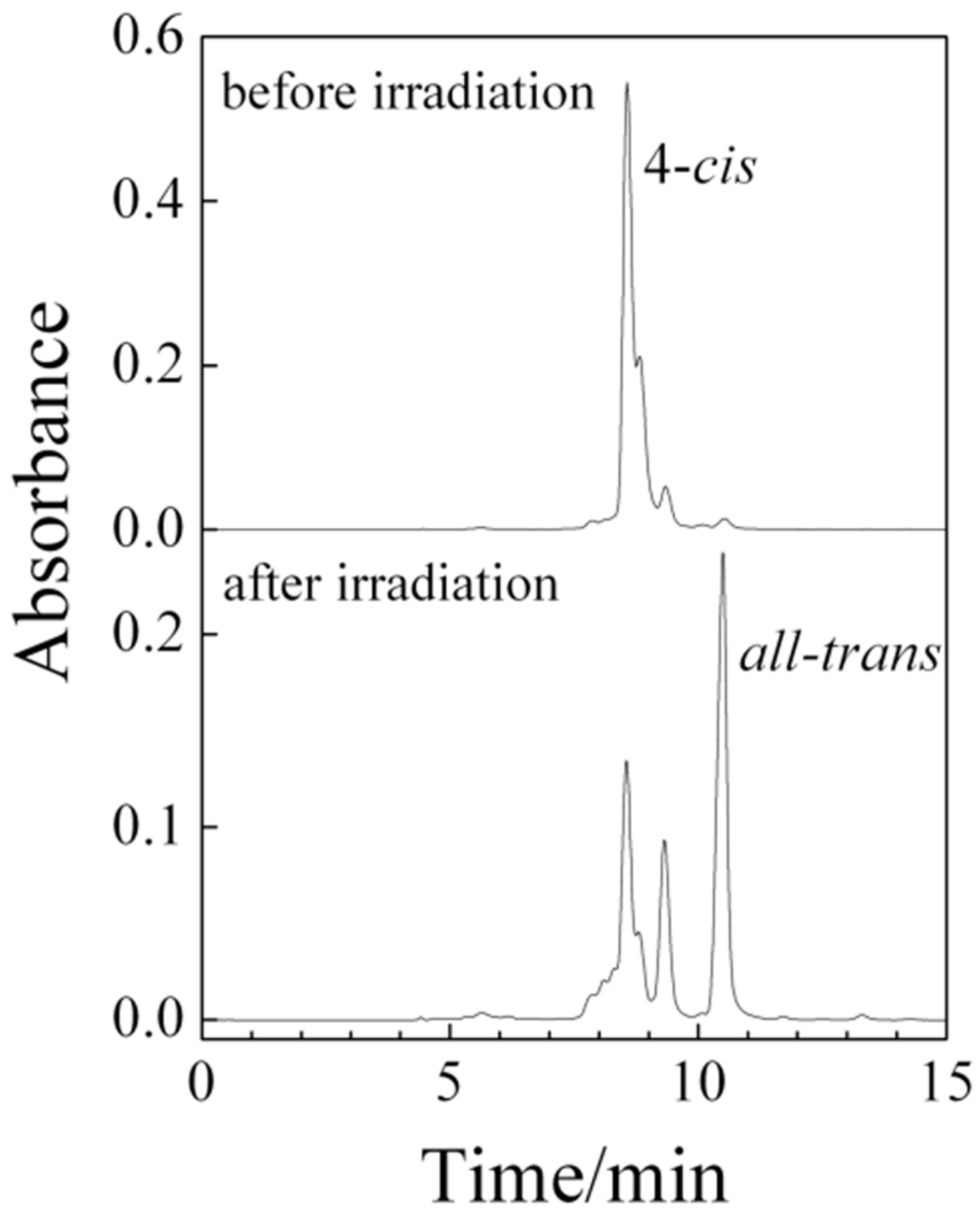

Figure 1.

HPLC of hexadecaheptaene before and after $396 \mathrm{~nm}$ irradiation. Chromatography was done on a $\mathrm{C}_{18}$ reverse phase column using acetonitrile as the mobile phase. Absorbance was monitored at $396 \mathrm{~nm}$. 


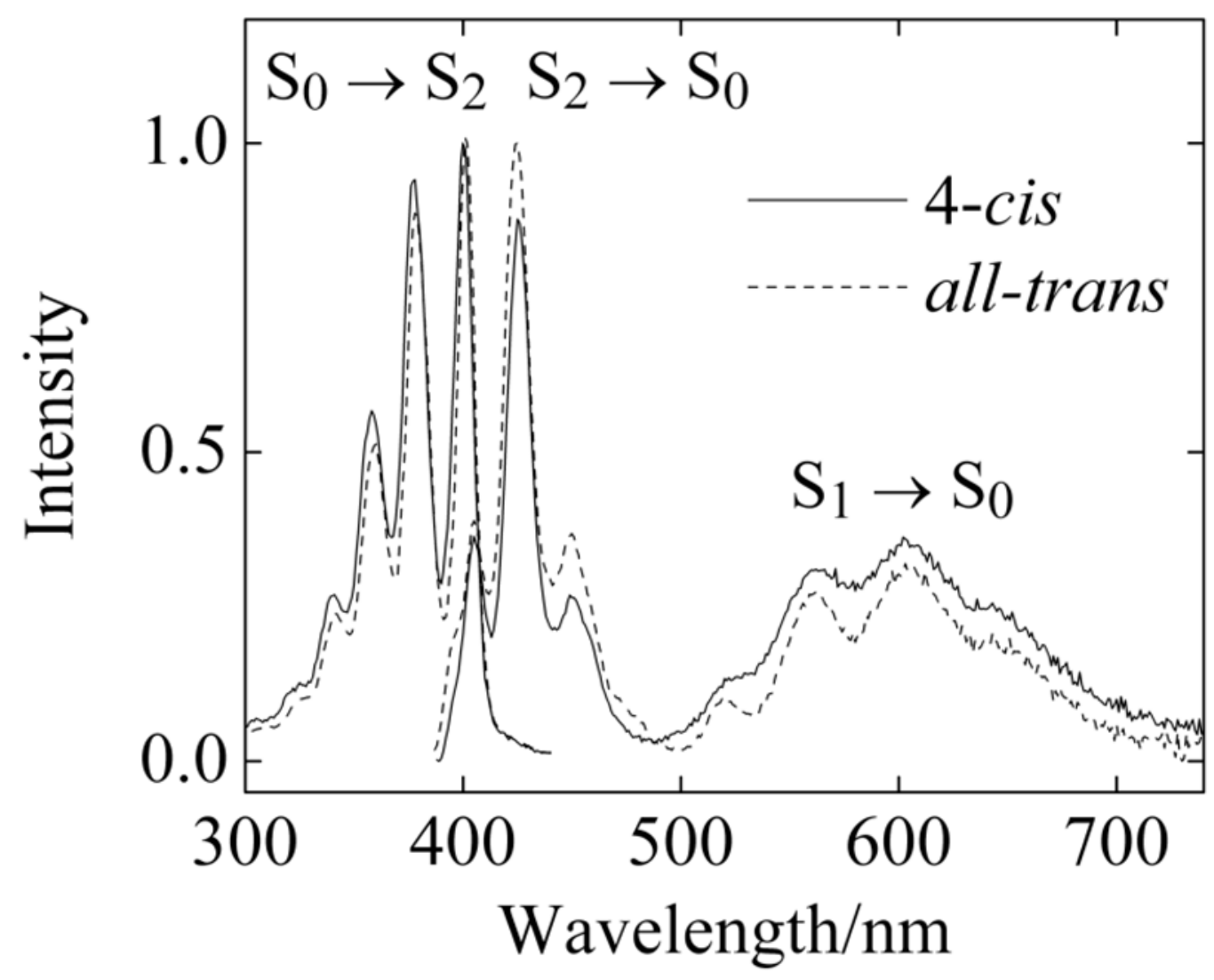

Figure 2.

Absorption and fluorescence spectra of 4-cis (378 nm excitation) and all-trans (377 nm excitation) hexadecaheptaene in $n$-pentadecane at room temperature. The absorption spectra are normalized to their maximum values. Maximum absorbances of $\sim 0.10$ minimized the effects of self-absorption. The fluorescence intensities have been corrected for the different room temperature absorbances of the two samples at the excitation wavelengths. Spectra were acquired with 1-nm bandpasses. 


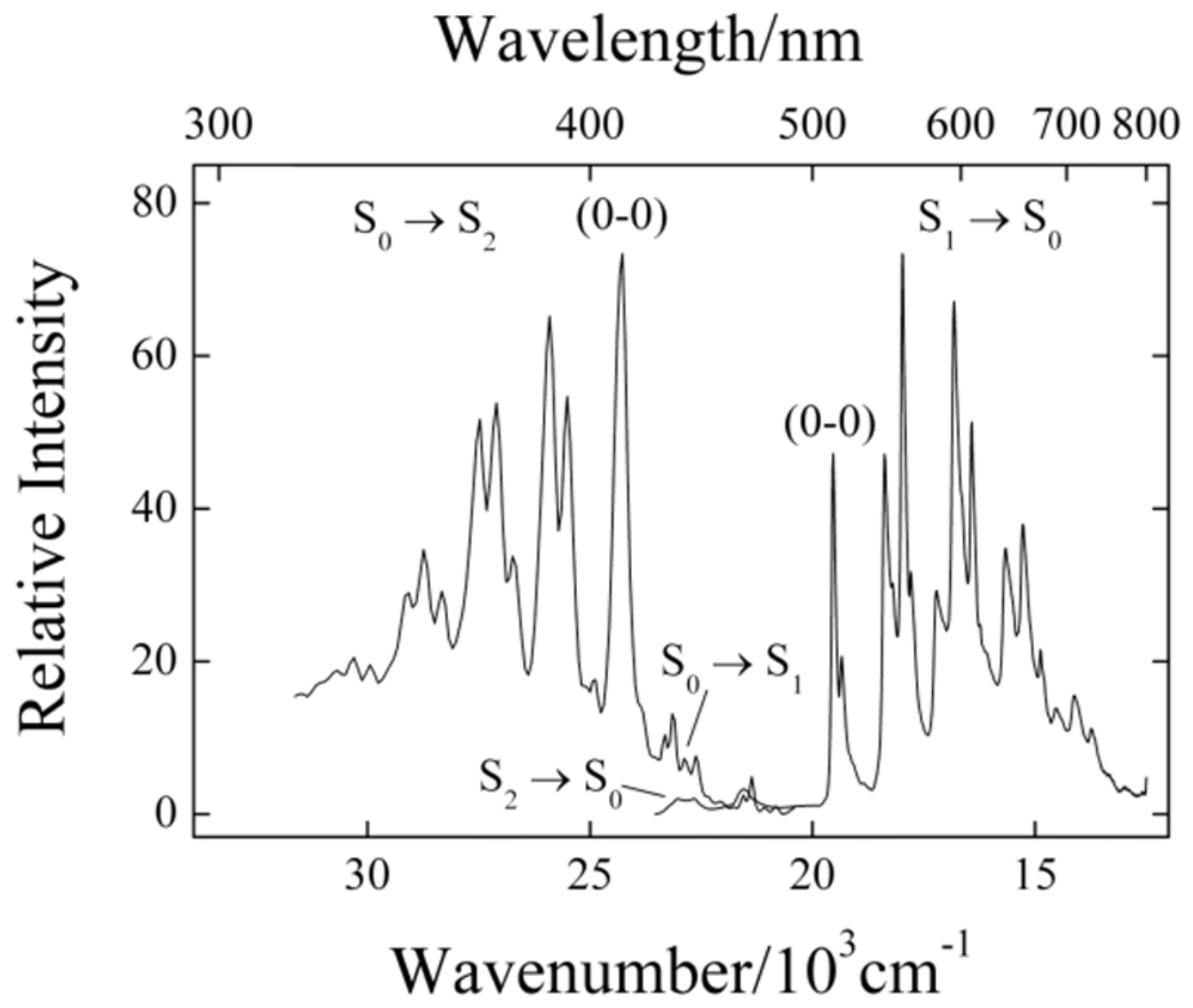

Figure 3.

Fluorescence excitation and emission spectra of 4-cis hexadecaheptaene in $n$-pentadecane at $77 \mathrm{~K}$. The fluorescence spectrum was obtained by exciting at $411 \mathrm{~nm}$, and the fluorescence excitation spectrum was monitored at $594 \mathrm{~nm}$. Maximum absorbances of $\sim 0.10$ minimized the effects of self-absorption. Fluorescence intensities were corrected for the different room temperature absorbances of the two samples at the excitation wavelengths. Spectra were acquired with 1-nm bandpasses. 

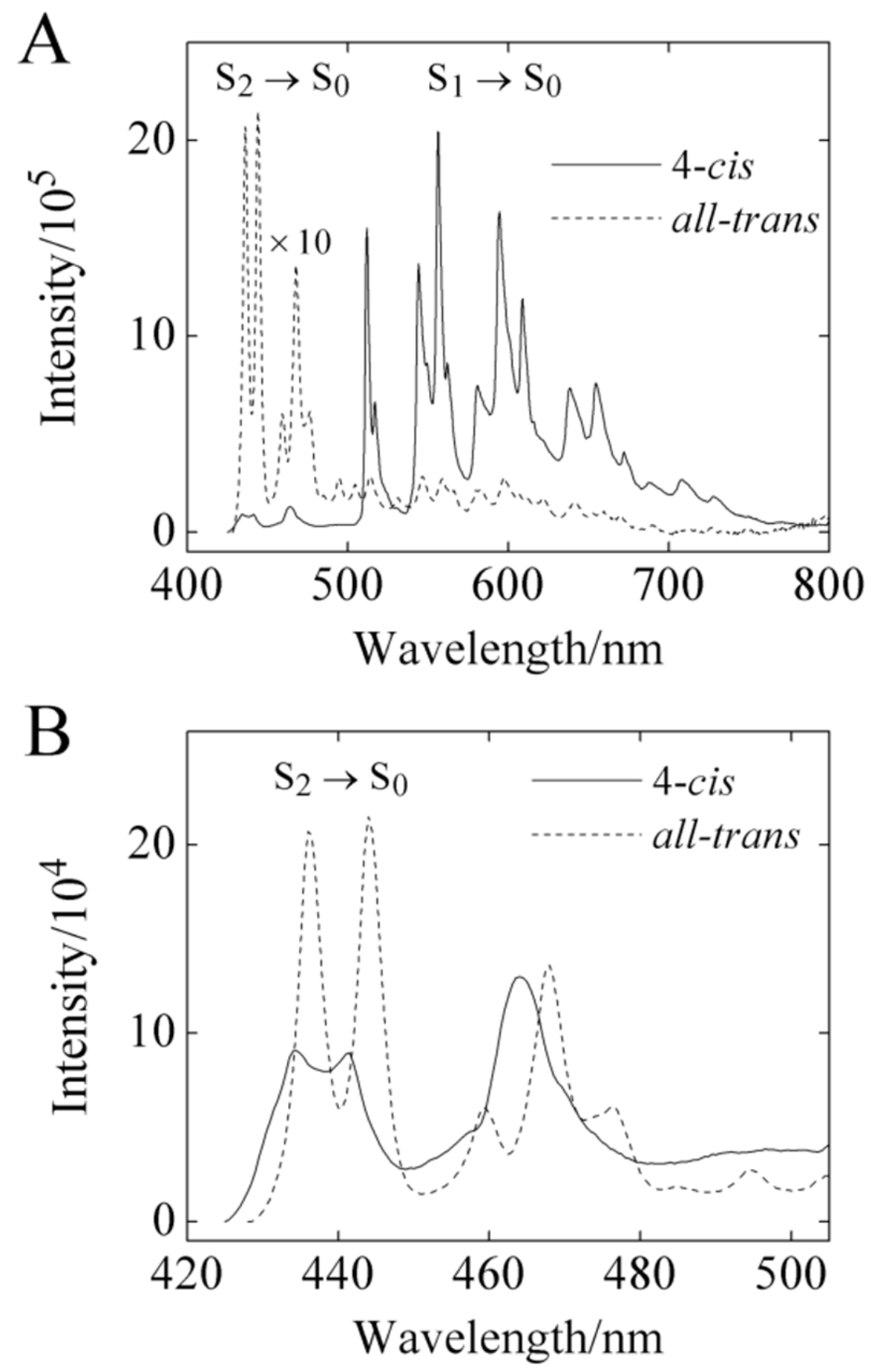

Figure 4.

A) Comparison of fluorescence spectra of 4-cis and all-trans hexadecaheptaene in $n$ pentadecane at $77 \mathrm{~K}$. The relative fluorescence intensities have been corrected for the difference in the room temperature absorbances of the two samples. Emission spectra were obtained by exciting into the $\mathrm{S}_{0} \rightarrow \mathrm{S}_{2}$ (0-0) bands (414 $\mathrm{nm}$ for all-trans, $411 \mathrm{~nm}$ for 4-cis). B) Expanded view of (A) showing the $S_{2} \rightarrow S_{0}$ fluorescence spectra of 4-cis hexadecaheptaene and alltrans hexadecaheptaene in $n$-pentadecane at $77 \mathrm{~K}$. Emission bandpasses were $1 \mathrm{~nm}$ for all spectra. 


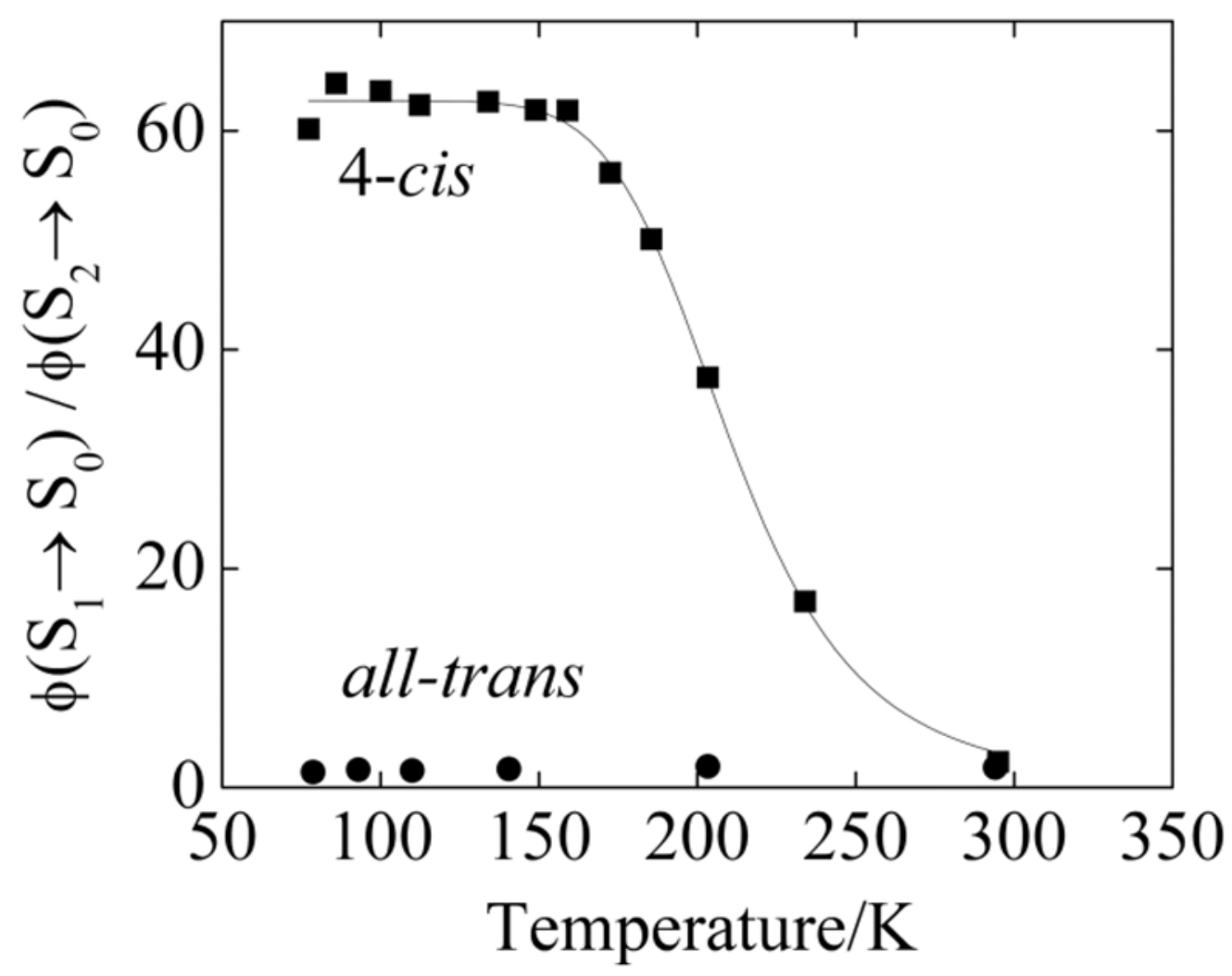

Figure 5.

Ratios of integrated fluorescence yields $\left(\varphi\left(\mathrm{S}_{1} \rightarrow \mathrm{S}_{0}\right) / \varphi\left(\mathrm{S}_{2} \rightarrow \mathrm{S}_{0}\right)\right)$ for 4-cis- (squares) and alltrans (circles) hexadecaheptaene as a function of temperature. The temperature dependence of the ratio for the 4-cis isomer was fit to Equation 1 assuming that $\mathrm{k}_{\mathrm{r}}, \mathrm{k}_{\mathrm{nr}}$, and $\varphi\left(\mathrm{S}_{2} \rightarrow \mathrm{S}_{0}\right)$ are independent of temperature. The four-parameter fit (solid line) gives $\mathrm{E}_{\mathrm{a}}=4.3 \pm 1.4 \mathrm{kcal}$. 


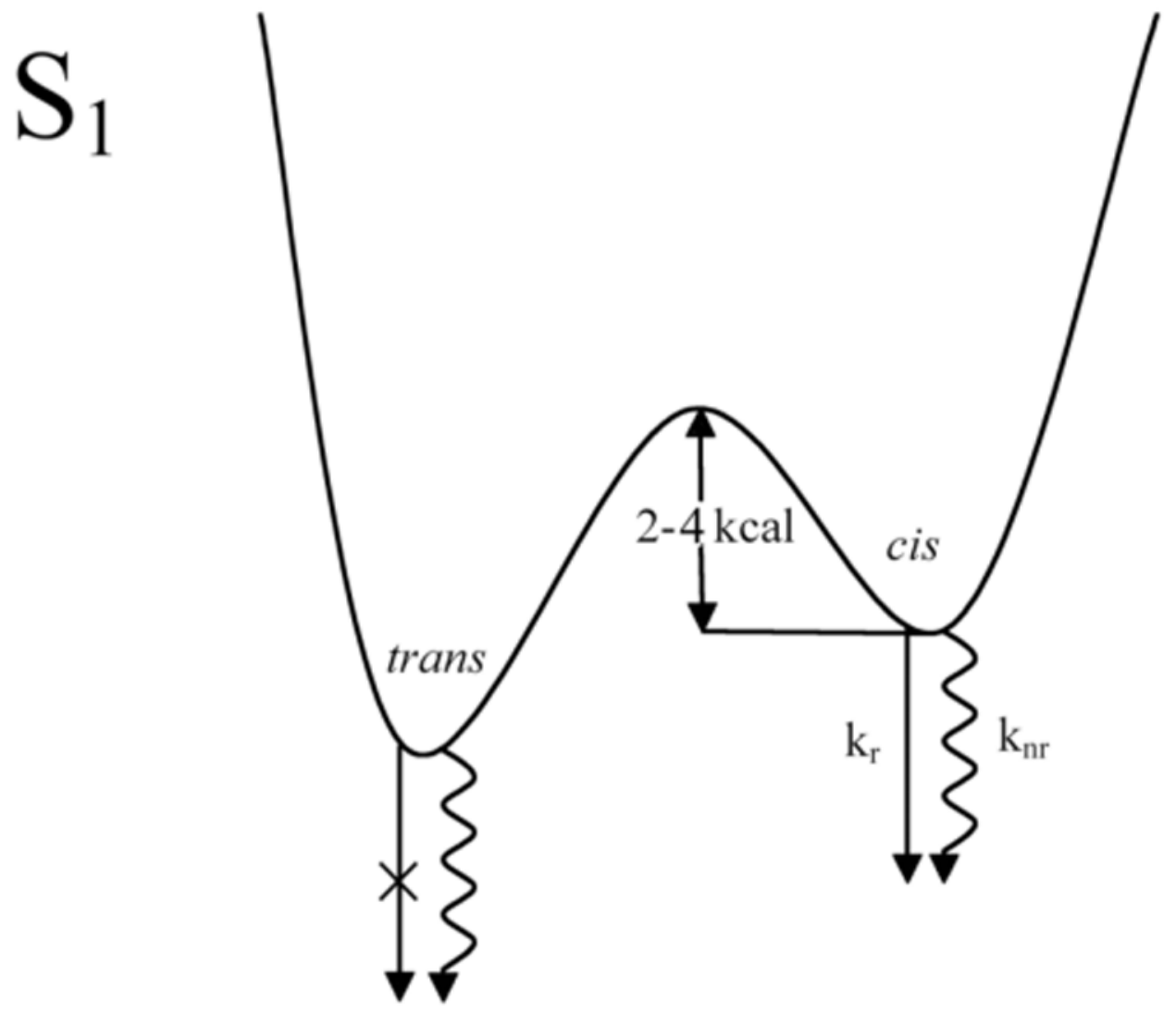

Figure 6.

Schematic potential energy surface for hexadecaheptaene in the $S_{1}\left(2^{1} \mathrm{~A}_{\mathrm{g}}{ }^{-}\right)$state. 


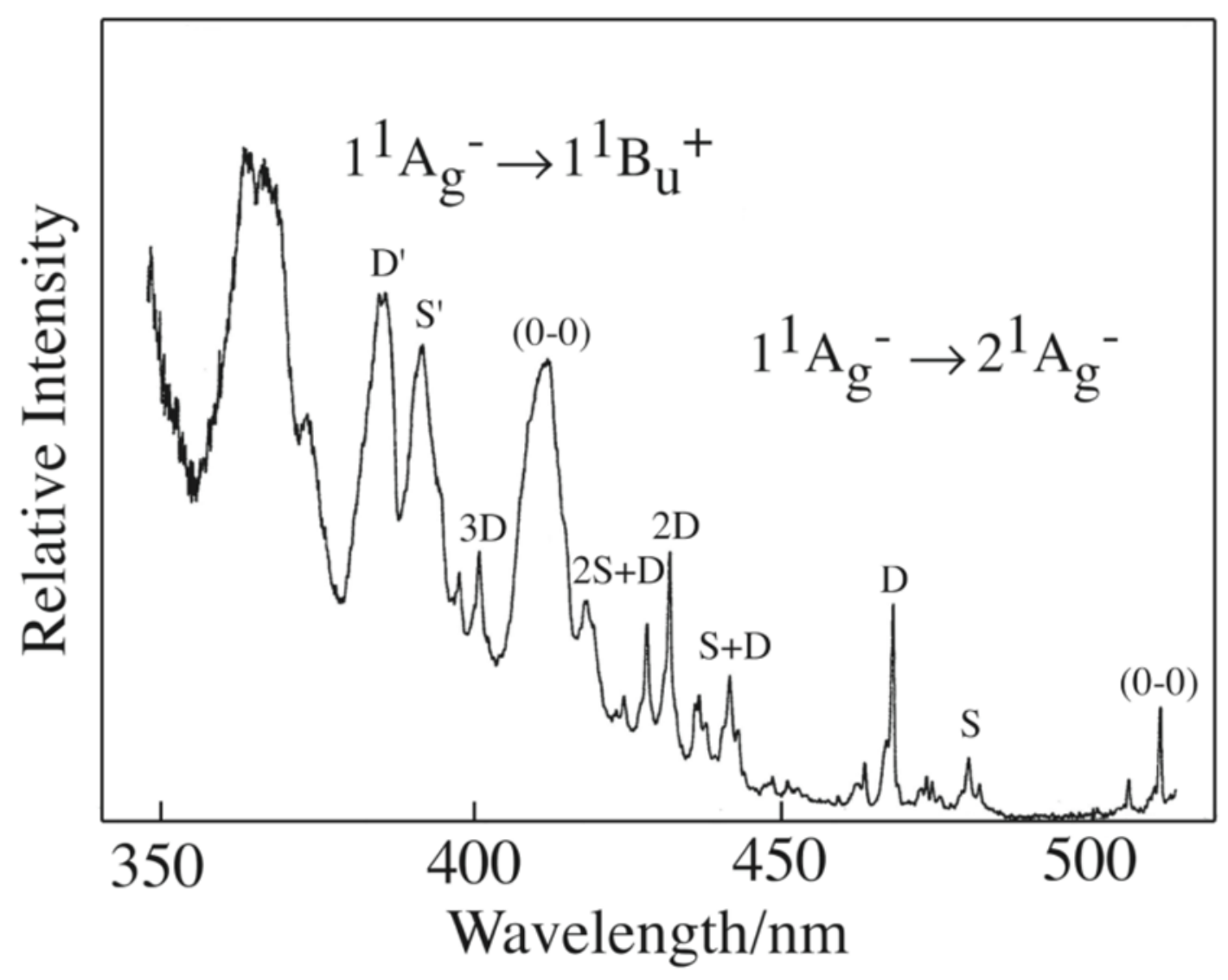

Figure 7.

Fluorescence excitation spectra $\left(1^{1} \mathrm{~A}_{\mathrm{g}}{ }^{-} \rightarrow 2^{1} \mathrm{~A}_{\mathrm{g}}{ }^{-}\right.$and $\left.1^{1} \mathrm{~A}_{\mathrm{g}}{ }^{-} \rightarrow 1^{1} \mathrm{~B}_{\mathrm{u}}{ }^{+}\right)$of 4cis-2,4,6,8,10,12,14-hexadecaheptaene in $10 \mathrm{~K} n$-pentadecane. Fluorescence was detected at $560 \mathrm{~nm}$. Vibronic features labeled with $\mathrm{S}$ and $\mathrm{D}$ (and $\mathrm{S}^{\prime}$ and $\mathrm{D}^{\prime}$ ) indicate combinations of C-C and $\mathrm{C}=\mathrm{C}$ symmetric stretching modes. This spectrum was obtained on a more concentrated sample than that used for the excitation spectrum presented in Fig. 3. This amplifies the $1^{1} \mathrm{~A}_{\mathrm{g}}{ }^{-} \rightarrow 2^{1} \mathrm{~A}_{\mathrm{g}}{ }^{-}$absorption. The excitation spectrum is not corrected for the wavelength dependence of the excitation system. (Figure adapted by the author, RLC, from Figure 3 of Reference 18). 